\title{
Stability of BTZ black strings
}

\author{
Lihui Liu and Bin Wang* \\ Department of Physics, Fudan University, Shanghai 200433, China
}

\begin{abstract}
We study the dynamical stability of the BTZ black string against fermonic and gravitational perturbations. The BTZ black string is not always stable against these perturbations. There exist threshold values for $\mathrm{m}^{2}$ related to the compactification of the extra dimension for fermonic perturbation, scalar part of the gravitational perturbation and the tensor perturbation, respectively. Above the threshold values, perturbations are stable; while below these thresholds, perturbations can be unstable. We find that this non-trivial stability behavior qualitatively agrees with that predicted by a thermodynamical argument, showing that the BTZ black string phase is not the privileged stable phase.

PACS numbers: $04.30 . \mathrm{Nk}, 04.70 . \mathrm{Bw}$
\end{abstract}

\section{INTRODUCTION}

Stability of gravitational configurations has been an intriguing subject of discussions since long. More recently, the discussion has been focused on the higher-dimensional black brane solutions provided by string theories. Gregory and Laflamme have shown in their pioneering work that neutral black strings in more than four dimensions suffer from a long-wavelength instability (GL instability) [1, 3]. In the last few years there have been a lot of progress achieved in understanding various aspects of the Gregory-Laflamme instability such as the GL instability with the presence of charge [2], and the GL instability in the $d S / A d S$ spacetimes [3, 4]. A review on the black string instability and whole list of relevant references are presented in [5].

Besides dynamical stability, there are also thermodynamical stability problems of black holes. However for usual black holes, such as Schwarzschild holes etc, they are thermodynamically unstable but dynamically stable against perturbations of metric. The profound connection between dynamical stability and thermodynamical stability was observed in black branes in supergravity. Gubser and Mitra proposed a correlated stability conjecture (GM conjecture) arguing that gravitational systems with translational symmetry lack stability against small perturbations often also lack thermodynamical stability [6]. Supports on this conjecture have been presented in [4, 7], while a set of counterexamples has also been raised [8]. It is of interest to examine this link further in various configurations. Recently a non-trivial relation between dynamical and thermodynamical properties in the background of charged Kaluza-Klein black hole with squashed horizons was discussed in [9].

In this work we are going to study the classical stability of the BTZ black string solution obtained by starting with a metric describing an accelerating black hole in $A d S_{4}$,

$$
\begin{gathered}
d s^{2}=\frac{1}{A^{2}(x-y)^{2}}\left[P(y) d t^{2}-\frac{d y^{2}}{P(y)}+\frac{d x^{2}}{Q(x)}+Q(x) d \varphi^{2}\right] ; \\
P(y)=-\lambda+k y^{2}-2 \bar{m} A y^{3}, Q(x)=1+k x^{2}-2 \bar{m} A x^{3},
\end{gathered}
$$

which satisfies the Einstein equation with a negative cosmological constant

$$
R_{A B}=-\left(3 / l_{4}^{2}\right) g_{A B},
$$

and $l_{4}=1 /(A \sqrt{\lambda+1}), A$ being the acceleration parameter. The spacetime is sliced onto the branes of $x / y=$ const., and the visible brane is situated at $x=0$. There are two configurations of interest:

\footnotetext{
*Electronic address: wangb@fudan.edu.cn
} 
(i) The black string phase. To orient with $\bar{m}=0$, the BTZ black string in $A d S_{4}$ can be obtained through coordinate transformation [10], which is described by

$$
d s^{2}=l_{4}^{2} d z^{2}+l_{4}^{2} a^{2}(z)\left[-H^{2} d t^{2}+H^{-2} d r^{2}+r^{2} d \varphi^{2}\right],
$$

where $H^{2}(r)=\lambda r^{2}-k$ and $a(z)=\sqrt{\lambda} \cosh z$ being the warp factor. We have the interest on $\lambda>0$ where locally the constant $z$ slices have the geometry of $A d S_{3}$. If $k=+1$, surfaces of constant $z$ have the geometry of BTZ black hole [11] described by

$$
d s_{3}^{2}=-\left(\lambda r^{2}-k\right) d t^{2}+\left(\lambda r^{2}-k\right)^{-1} d r^{2}+r^{2} d \varphi^{2},
$$

which is the solution of $R_{\mu \nu}=-2 \lambda g_{\mu \nu}$.

Here, Greek letters take the values 0,1,2 describing the brane and the capital Latin letters take the values 0,1,2,3 describing the bulk, where 3 indicates the extra dimension (i.e. $x^{0}=t, x^{1}=r, x^{2}=\varphi, x^{3}=z$ ).

(ii) The black hole phase. Turn to the case with $m>0, Q(x)$ has only one positive root $x_{2}$. Taking $\Delta \varphi=$ $4 \pi /\left|Q^{\prime}\left(x_{2}\right)\right|$, the conical singularity can be avoided. The black hole localized on a brane is described by [10]

$$
d s^{2}=\frac{1}{A^{2}}\left[-\left(\lambda r^{2}-k-\frac{2 \bar{m} A}{r}\right) d t^{2}+\left(\lambda r^{2}-k-\frac{2 \bar{m} A}{r}\right)^{-1} d r^{2}+r^{2} d \varphi^{2}\right],
$$

which is the BTZ-like black hole, with extra terms of the form $2 \bar{m} A / r$ coming from the four-dimensional nature of the black hole. If $2 \bar{m} A \ll \lambda^{-1 / 2}$, the extra term is negligible outside the horizon, and so the exterior geometry is essentially identical to that of a BTZ black hole [10].

A global thermodynamical argument using the entropy was presented to argue about the stability of these two configurations [10]. For the BTZ black string, it was shown that its thermodynamic mass $M_{4}$ precisely agrees with that of the projected BTZ black hole $M_{3}$. The four-dimensional entropy of the black string was also found in agreement with the three dimensional entropy

$$
S_{B S}=\frac{\pi}{A} \sqrt{\frac{2 M_{3}}{\lambda G_{3}}}
$$

where $M_{3}$ is the projected mass of the BTZ black string on the visible brane. The black hole entropy was obtained in the form

$$
S_{B H}=\frac{2 \pi}{G_{4} A^{2}} \frac{\tilde{z}}{\lambda+3 \tilde{z}^{2}+2 \tilde{z}^{3}}
$$

The auxiliary variable $\tilde{z}$ is introduced by the identity $2 \bar{m} A=\frac{\left(\lambda+\tilde{z}^{2}\right) \tilde{z} \sqrt{1+\tilde{z}}}{\left(\lambda-\tilde{z}^{3}\right)^{3 / 2}}$ and noting that $\tilde{z}$ is a monotonic function of $\bar{m} A$, which leads to $\bar{m} A \rightarrow 0, \tilde{z} \approx 2 \bar{m} A \sqrt{\lambda} ; \bar{m} A \rightarrow \infty, \tilde{z} \approx \lambda^{1 / 3}$.

Equating the black hole and black string entropies, Eq.(7) and Eq.(8), and identifying the mass of the two phases by substituting $M_{3}=\frac{1}{2 G_{3}} \frac{\tilde{z}^{2}(1+\tilde{z})\left(\lambda-\tilde{z}^{3}\right)}{\left(\lambda+3 \tilde{z}^{2}+2 \tilde{z}^{3}\right)^{2}}$, we get a fourth order polynomial in terms of the auxiliary variable $\tilde{z}\left(\lambda-\tilde{z}^{2}-\tilde{z}^{3}\right)=$ 0 . The root $\tilde{z}=0$ simply indicates that the mass and entropy of the localized BTZ black hole (when $\bar{m} A \rightarrow 0$ ) match that of the black string, which shows that thermodynamically there is no privileged stable phase in these two configurations. When $\bar{m} A$ increases so that the auxiliary variable $\tilde{z}$ reaches $\tilde{z}_{x}$, which satisfies $\tilde{z}_{x}^{2}+\tilde{z}_{x}^{3}=\lambda$, the black hole and the black string entropies equal to each other again. Except these two points, the entropy comparison of black hole and black string phase is not illuminating in the auxiliary variable $\tilde{z}$. However expressing the entropies of the black hole and the black string phase in terms of their masses, it was shown in Fig.1 of [10] that, for small $\bar{m} A$, the black string phase entropy exceeds that of the localized black hole, while for large $\bar{m} A$, the result goes in the contrary; the changeover point is at the root $\tilde{z}_{x}$.

In the present work, we will study the stability of the BTZ black string against fermionic and gravitational perturbations and examine the relation between the dynamical stability against different fields' perturbations and the thermodynamical stability observed in [10]. Since we have, in this background, exact solutions for both the black strings in 3+1 dimensions as well as BTZ black holes on the brane in 2+1-dimensions, we can examine the stability of the black string in detail.

\section{FERMION PERTURBATION}

In this section we take a first look at the stability of the BTZ black string against fermionic perturbation. We assume that the Fermion field exists in the bulk. We will derive the fermionic perturbation equation and examine the mode which signals the stability of the black string. 


\section{A. Perturbation Equation}

For the massless Fermion field, it is described by the Dirac equation 12$]$

$$
\gamma^{a} e_{a}^{A}\left(\partial_{A}+\Gamma_{A}\right) \Psi=0 .
$$

Here $\gamma^{a}$ is the Dirac matrix satisfying $\left\{\gamma^{a}, \gamma^{b}\right\}=2 \eta^{a b}, \eta^{a b}$ is the Lorentz metric with the signature $(-1,1,1,1) . e_{a}^{A}$ is the tetrad so chosen that $e_{a}^{A} e_{b}^{B} \eta^{a b}=g^{A B}$, whose indices in small letters are raised or lowered by $\eta_{a b}$ and indices in capital letters are raised or lowered by $g_{A B} . \Gamma_{A}$ is the affinity of covariant derivative, defined by $\Gamma_{A}=\frac{1}{8}\left[\gamma^{a}, \gamma^{b}\right] e_{a}^{B} e_{b B ; A}$, $e_{b B ; A}=e_{b B, A}-\Gamma_{B A}^{E} e_{b E}$. In the spacetime background of Eq.(44), we can rewrite Eq.(9) as

$$
\frac{\gamma^{0}}{H} \partial_{t} \Psi+\gamma^{1} H\left[\partial_{r}+\frac{\left(2 \lambda r^{2}-k\right)}{2 r\left(\lambda r^{2}-k\right)}\right] \Psi+\frac{\gamma^{2}}{r} \partial_{\varphi} \Psi+\gamma^{3} a\left(\partial_{z}+\frac{3 \partial_{z} a}{2 a}\right) \Psi=0 .
$$

Separating the wave function by

$$
\Psi(t, r, \varphi, z)=R(r) Z(z) e^{-i \omega t+i \mu \varphi}
$$

and taking

$$
\begin{aligned}
& R(r)=\left[r^{2}\left(\lambda r^{2}-k\right)\right]^{-1 / 4} \Phi(r), \\
& Z(z)=\exp \left[-\int \frac{3 \partial_{z} a}{2 a} d z+i m \int \frac{d z}{a}\right],
\end{aligned}
$$

we can obtain the radial wave equation in the form

$$
-i \omega H^{-1} \gamma^{0} \Phi+H \gamma^{1} \partial_{r} \Phi+i\left(\mu r^{-1} \gamma^{2}+m \gamma^{3}\right) \Phi=0 .
$$

Here, $\mu$ takes the value of (integer times $2 \pi) /($ period of $\varphi$ ) as required by the periodical condition in $\varphi$. The value of $m$ is determined by the compactification of the extra-dimension.

Choosing

$$
\gamma^{0}=\left(\begin{array}{cc}
0 & -i \\
-i & 0
\end{array}\right), \quad \gamma^{i}=\left(\begin{array}{cc}
0 & -i \sigma^{i} \\
i \sigma^{i} & 0
\end{array}\right), \quad i=1,2,3
$$

and letting $\Phi=\left(\phi_{1}, \phi_{2}\right)^{\mathrm{T}}, \phi_{a}=\left(\phi_{a+}, \phi_{a-}\right)^{\mathrm{T}}(a=1,2)$, we have

$$
\begin{aligned}
i \omega H^{-1} \phi_{1}+H \sigma^{1} \partial_{r} \phi_{1}+i\left(\mu r^{-1} \sigma^{2}+m \sigma^{3}\right) \phi_{1} & =0 \\
-i \omega H^{-1} \phi_{2}+H \sigma^{1} \partial_{r} \phi_{2}+i\left(\mu r^{-1} \sigma^{2}+m \sigma^{3}\right) \phi_{2} & =0 .
\end{aligned}
$$

We focus on the second equation and the first one can be solved easily by changing $\omega \rightarrow-\omega$. Explicitly writing out the Pauli matrices, Eq.(17) gives

$$
\left\{\begin{array}{l}
\left(-i \omega H^{-1}+i m\right) \phi_{2+}+\left(H \partial_{r}-\mu r^{-1}\right) \phi_{2-}=0 \\
\left(-i \omega H^{-1}-i m\right) \phi_{2-}+\left(H \partial_{r}+\mu r^{-1}\right) \phi_{2+}=0
\end{array}\right.
$$

Defining $Y_{ \pm}=\left(\frac{r m+i \mu}{r m-i \mu}\right)^{ \pm 1 / 4}\left(\phi_{2+} \pm \phi_{2-}\right)$, we can change these equations into

$$
\left(\partial_{\bar{r}} \mp S\right) Y_{ \pm} \pm i m Y_{\mp}=0,
$$

where

$$
\begin{aligned}
\bar{r}= & \int \frac{\sqrt{r^{2} m^{2}+\mu^{2}}}{m r H} d r \\
S(r) & =\frac{i m r}{H \sqrt{r^{2} m^{2}+\mu^{2}}}\left[\omega-\frac{\mu m H^{2}}{2\left(r^{2} m^{2}+\mu^{2}\right)}\right] .
\end{aligned}
$$

Decoupling Eqs.(19), we have

$$
-\partial_{\bar{r}}^{2} Y_{ \pm}+\left(m^{2}+S^{2} \pm \partial_{\bar{r}} S\right) Y_{ \pm}=0
$$


Eq.(19) and Eq.(22) allow us to look at the asymptotic behavior of $Y_{ \pm}$at the horizon $r=r_{H}=\sqrt{k / \lambda}$ and at spatial infinity $r \rightarrow \infty$. In the vicinity of the horizon $r \rightarrow r_{H}+0, Y_{ \pm} \sim\left(r-r_{H}\right)^{\rho_{ \pm}}$, and from Eq.(22) we require $\rho_{ \pm}$to satisfy $2 \rho_{ \pm}^{2}-\rho_{ \pm}+\omega^{2} / 2 \lambda k \pm i \omega / 2 \sqrt{\lambda k}=0$. Since there exist only ingoing waves near the horizon, we have $\rho_{+}=1 / 2-i \omega / 2 \sqrt{\lambda k}$, and $\rho_{-}=-i \omega / 2 \sqrt{\lambda k}$ and thus in the limit $r \rightarrow r_{H}+0$,

$$
Y_{+} \sim\left(r-r_{H}\right)^{\frac{1}{2}-\frac{i \omega}{2 \sqrt{\lambda k}}}, \quad Y_{-} \sim\left(r-r_{H}\right)^{-\frac{i \omega}{2 \sqrt{\lambda k}}}
$$

For a consistency check this result also satisfies the coupled first-order equation (19). Similarly we let $r \rightarrow \infty$ and find asymptotic solutions satisfying Eq.(19) and Eq.(22)

$$
Y_{ \pm} \sim\left\{\begin{array}{c}
r^{m / \sqrt{\lambda}} \\
r^{-m / \sqrt{\lambda}}
\end{array}\right\}, r \rightarrow \infty .
$$

\section{B. Quasinormal Frequencies of Fermionic Perturbation}

We now investigate the stability problem by solving Eq.(19), which is similar to the quasinormal modes (QNMs) study (a review on the QNMs study can be found in [13, 14]).

In $A d S$ spacetime, the QNMs were defined in [17] by only ingoing modes near the horizon while at the spatial infinity the wave function $\Psi$, here equivalent to $r^{-1} Y_{ \pm}$, vanishes. This boundary condition allows only a discrete set of complex $\omega$ to exist. If the imaginary part of the QNM frequency is negative, we will have the decaying mode. However, if the imaginary part of QNM frequency is positive, we will have a growing mode, indicating that the spacetime is unstable against the field perturbation.

To calculate the QNM frequencies, we need to solve Eq.(19) and select only solutions which satisfy $\Psi \rightarrow 0$ as $r \rightarrow \infty$. For simplicity, we will let $\mu=0$, which allows to solve Eq. (19) analytically. Working with the decoupled equation Eq.(22), we can rewrite them as

$$
H^{2} \partial_{r}^{2} Y_{-}+\lambda r \partial_{r} Y_{-}+\left(m^{2}-H^{2}+i \omega H^{-2} \lambda r\right) Y_{-}=0
$$

This equation is symmetric under the transformation of $r \rightarrow \alpha r, m \rightarrow m / \alpha$ and $\omega \rightarrow \omega / \alpha$. In the following we will set $\lambda=1$. Taking $x=(r-1) /(r+1), Y_{-}=x^{-i \omega / 2}(x-1)^{-m} y(x)$, Eq.(25) becomes a hypergeometric equation (see also [18])

$$
x(1-x) \frac{d^{2} y}{d x^{2}}+[c-(1+a+b) x] \frac{d y}{d x}-a b y=0
$$

where $a=-m, b=1 / 2-i \omega-m$, and $c=1 / 2-i \omega$. We choose its solution to be simply ${ }_{2} F_{1}(a, b, c ; x)$, and eliminate the other one $x^{1-c}{ }_{2} F_{1}(a-c+1, b-c+1,2-c ; x)$, so that $Y_{-}$contains only ingoing modes near the horizon embodied by the factor $x^{-i \omega / 2}$. Therefore $Y_{-}=x^{-i \omega}(x-1)^{-m}{ }_{2} F_{1}(a, b, c ; x)$. Considering ${ }_{2} F_{1}(a, b, c ; x)=$ $(1-x)^{c-a-b}{ }_{2} F_{1}(c-a, c-b, c ; x)$ [15], we can rewrite the solution as

$$
Y_{-}=x^{-i \omega / 2}(x-1)^{-|m|}{ }_{2} F_{1}(\tilde{a}, \tilde{b}, c ; x),
$$

where $\tilde{a}=-|m|$ and $\tilde{b}=1 / 2-i \omega-|m|$ satisfying $\operatorname{Re}(c-\tilde{a}-\tilde{b})=2|m|>0$. We need to substitute Eq.(27) into the first equation of Eqs.(19) to get the explicit expression of $Y_{+}$. Using $\frac{d}{d x}{ }_{2} F_{1}(a, b, c ; x)=\frac{a b}{c}{ }_{2} F_{1}(a+1, b+1, c+1 ; x)$ [15], we have

$$
Y_{+}=-i \epsilon x^{1 / 2-i \omega / 2}(x-1)^{-|m|}\left[{ }_{2} F_{1}(\tilde{a}, \tilde{b}, c ; x)+C(x-1){ }_{2} F_{1}(\tilde{a}+1, \tilde{b}+1, c+1 ; x)\right],
$$

where $\epsilon$ is the sign of $m$ and $C=\frac{1-2 i \omega-|m|}{1-2 i \omega}$. Using the transformation

$$
\begin{aligned}
& { }_{2} F_{1}(a, b, c ; x)=\frac{\Gamma(c) \Gamma(c-a-b)}{\Gamma(c-a) \Gamma(c-b)}{ }_{2} F_{1}(a, b, a+b-c+1 ; 1-x) \\
& \quad+(1-x)^{c-a-b} \frac{\Gamma(c) \Gamma(a+b-c)}{\Gamma(a) \Gamma(b)}{ }_{2} F_{1}(c-a, c-b, c-a-b+1,1-x),
\end{aligned}
$$

and the $x=\frac{r-1}{r+1}$ or $x-1 \sim-2 / r$ at spatial infinity, we can write the asymptotical form of $Y_{ \pm}$at spatial infinity as

$$
\begin{aligned}
& Y_{+} \sim i \epsilon\left(D_{+} r^{|m|}+D_{-} r^{-|m|}\right) \\
& Y_{-} \sim D_{+} r^{|m|}+D_{-} r^{-|m|}
\end{aligned}
$$


where

$$
D_{+}=(-2)^{-|m|} \frac{\Gamma(c) \Gamma(c-\tilde{a}-\tilde{b})}{\Gamma(c-\tilde{a}) \Gamma(c-\tilde{b})}, \quad D_{-}=(-2)^{|m|} \frac{\Gamma(c) \Gamma(\tilde{a}+\tilde{b}-c)}{\Gamma(\tilde{a}) \Gamma(\tilde{b})} .
$$

This matches the asymptotical behavior already obtained in Eq.(24).

When $|m|<\sqrt{\lambda}$, it is easy to see that the boundary condition $r^{-1} Y_{ \pm} \rightarrow 0$ at spatial infinity can be satisfied automatically, thus we have no limit from the boundary condition requirement on the value of $\omega$. This is quite unusual since the QNM with any frequency, even with the positive imaginary part can be allowed. This implies that when $|m|<\sqrt{\lambda}$, the perturbation may have growing modes and the black string is unstable against the Fermonic perturbation.

On the other hand for the perturbations of $|m| \geq \sqrt{\lambda}$, the fulfillment of boundary condition $r^{-1} Y_{ \pm} \rightarrow 0$ at spatial infinity calls for the condition that $D_{+}=0$. Thus we need to let $c-\tilde{a}$ be zero or negative integer, which gives the discrete values of QNM frequencies

$$
\omega=-i(1 / 2+n+|m| / \sqrt{\lambda}), \quad n=0,1,2, \ldots
$$

where we have recovered $\lambda$. The purely negative imaginary frequencies indicate that when $|m| \geq \sqrt{\lambda}$ the perturbation of massless Dirac field exhibits the decay mode showing that the black string is stable under such perturbation.

\section{GRAVITATIONAL PERTURBATION}

Now we consider the linear perturbation of the BTZ black string spacetime, which we will denote as $g_{A B}+h_{A B}$ where $g_{A B}$ stands for the components of the unperturbed black string metric and $h_{A B}$ is the metric perturbation.

\section{A. Perturbation equations}

The perturbation equations can be greatly simplified by choosing appropriate gauge conditions, which was discussed in detail in studying the gravitational wave in asymptotically flat spacetimes (e.g., [16], §4.4). We will employ gauge conditions on $h_{A B}$ including the tracelessness condition, the transversality condition, namely $h_{A}^{A}=0, h_{; B}^{A B}=0$, and $h_{4 \mu}=0$, so that

$$
h_{A B}(t, r, \varphi, z)=e^{\Omega t-i \mu \varphi}\left(\begin{array}{cccc}
H_{t t}(r, z) & H_{t r}(r, z) & H_{t \varphi}(r, z) & 0 \\
H_{r t}(r, z) & H_{r r}(r, z) & H_{r \varphi}(r, z) & 0 \\
H_{\varphi t}(r, z) & H_{\varphi r}(r, z) & H_{\varphi \varphi}(r, z) & 0 \\
0 & 0 & 0 & H_{z z}(r, z)
\end{array}\right) .
$$

The linearized Ricci tensor has the form $\delta R_{B D}=-\frac{1}{2} \square^{(4)} h_{B D}+R_{E(B} h_{D)}^{E}-R_{B C D E} h^{C E}$. Applying this to linearize Eq.(3), we get

$$
\square^{(4)} h_{B D}+2 R_{B C D E} h^{C E}=0 .
$$

Inserting the perturbation Eq.(34) into the above equation, we find immediately that with the trace-free condition, the equation governing $H_{z z}$ is decoupled from the rest, giving the description of scalar part of the perturbation. Taking the ansatz $H_{z z}(r, z)=H_{z z}(r) u_{m}(z)$, we have

$$
\left(\lambda r^{2}-k\right) H_{z z, r r}+r^{-1}\left(3 \lambda r^{2}-k\right) H_{z z, r}-\left[\left(\lambda r^{2}-k\right)^{-1} \Omega^{2}-2 \lambda+\mu^{2} r^{-2}+m^{2}\right] H_{z z}=0
$$

where $m^{2}$ is the effective mass of the Kaluza-Klein modes satisfying

$$
a^{-1}\left(a^{3} u_{m}^{\prime}\right)^{\prime}-6\left(a^{\prime}\right)^{2} u_{m}+m^{2} u_{m}=0,
$$

where the prime denotes the derivative with respect to $z$. In the vicinity of the horizon and near the spatial infinity, solutions of Eq.(36) asymptotically have behaviors near the horizon and the spatial infinity

$$
\begin{aligned}
& H_{z z} \sim\left(r-r_{H}\right)^{ \pm \frac{\Omega}{2 \sqrt{\lambda k}}}, \quad r \rightarrow r_{H}, \\
& H_{z z} \sim r^{-1 \pm \sqrt{m^{2} / \lambda-1}}, \quad r \rightarrow \infty .
\end{aligned}
$$


$r_{H}=\sqrt{k / \lambda}$ denotes the event horizon. In order to have only ingoing wave near the horizon, we require the sign in the exponential of Eq.(38) to be positive.

Now we turn to discuss the tensor perturbation. Since $H_{z z}$ is independent of the rest, i.e., of the tensor perturbation, we set $H_{z z} \equiv 0$. However to obtain the perturbation equation, we need to take further simplification. Considering just the s-wave [1] that $H_{t \varphi}=0$ and $H_{r \varphi}=0$, we have

$$
h_{A B}(t, r, z)=e^{\Omega t}\left(\begin{array}{cccc}
H_{t t}(r, z) & H_{t r}(r, z) & 0 & 0 \\
H_{r t}(r, z) & H_{r r}(r, z) & 0 & 0 \\
0 & 0 & H_{\varphi \varphi}(r, z) & 0 \\
0 & 0 & 0 & 0
\end{array}\right) .
$$

Using the metric Eqs.(4) (5) and the perturbation in the form (40), we find that the perturbation equation reduces to

$$
a^{-2}\left(\square^{(3)} h_{\mu \nu}+2 R_{\mu \lambda \nu \rho}^{(3)} h^{\lambda \rho}\right)+a\left(a^{-1} h_{\mu \nu}^{\prime}\right)^{\prime}-2 a^{-1} a^{\prime \prime} h_{\mu \nu}=0
$$

where the prime denotes the derivative with respect to $z$, and the greek index relates to the three-dimensional spacetime Eq.(5). Separating the perturbation into $h_{\mu \nu}(t, r, z)=h_{\mu \nu}(t, r) v_{m}(z)=e^{\Omega t} H_{\mu \nu}(r) v_{m}(z)$, we can rewrite (41) into

$$
a\left(a^{-1} v_{m}^{\prime}\right)^{\prime}-2 a^{-1} a^{\prime \prime} v_{m}+a^{-2} m^{2} v_{m}=0
$$

and

$$
\square^{(3)} h_{\mu \nu}+2 R_{\mu \lambda \nu \rho}^{(3)} h^{\lambda \rho}-m^{2} h_{\mu \nu}=0 .
$$

With the aid of gauge conditions, we can obtain a second order differential equation of $H_{t r}$ from Eq. (43) through tedious calculations, which reads

$$
\begin{aligned}
& {\left[\Omega^{2}+\left(\lambda r^{2}-k\right) m^{2}+\lambda^{2} r^{2}-2 \lambda k\right] H_{t r, r r} } \\
+ & {\left[\frac{7 \lambda r^{2}-k}{r\left(\lambda r^{2}-k\right)} \Omega^{2}+\frac{5 \lambda r^{2}-k}{r} m^{2}+\frac{\lambda\left(5 \lambda^{2} r^{4}-13 \lambda k r^{2}+2 k^{2}\right)}{r\left(\lambda r^{2}-k\right)}\right] H_{t r, r} } \\
+ & {\left[\frac{\lambda\left(3 \lambda r^{2}-2 k\right)\left(\lambda^{2} r^{4}-4 \lambda k r^{2}-k^{2}\right)}{r^{2}\left(\lambda r^{2}-k\right)^{2}}+\frac{2 \lambda^{2} r^{4}-2 \lambda k r^{2}-k^{2}}{r^{2}\left(\lambda r^{2}-k\right)} m^{2}\right.} \\
+ & \left.\frac{6 \lambda^{2} r^{4}-k^{2}}{r^{2}\left(\lambda r^{2}-k\right)^{2}} \Omega^{2}-\left(m^{2}+\frac{\Omega^{2}}{\lambda r^{2}-k}\right)^{2}\right] H_{t r}=0 .
\end{aligned}
$$

Its asymptotic behaviors near the horizon and the spatial infinity have forms as

$$
\begin{aligned}
& H_{t r} \sim\left(r-r_{H}\right)^{-1 \pm \frac{\Omega}{2 \sqrt{\lambda k}}}, \quad r \rightarrow r_{H}, \\
& H_{t r} \sim r^{-2 \pm \sqrt{m^{2} / \lambda+1}, \quad r \rightarrow \infty .}
\end{aligned}
$$

Considering only the ingoing wave near the horizon, we will just keep the positive sign in the exponent of Eq.(45).

We have obtained equations describing the scalar part of the gravitational perturbation (36) and the tensor perturbation (44). In the following we will adopt the boundary condition that at the spatial infinity the wave functions $H_{z z}$ and $H_{t r}$ vanish so as to solve these equations and examine the stability of the BTZ black string against gravitational perturbations.

\section{B. Exact Solution of Scalar Part Perturbation}

Taking $H_{z z}(r)=r^{-1 / 2} \psi(r)$ and $\omega=i \Omega$, Eq.(36) can be put in the form of a Schrödinger equation,

$$
\frac{d^{2} \psi}{d r_{*}^{2}}+\left[\omega^{2}-V(r)\right] \psi=0
$$

where $r_{*}$ is the tortoise coordinate defined by $r_{*}=\int d r /\left(\lambda r^{2}-k\right)=\frac{1}{2 \sqrt{\lambda k}} \ln \frac{r-r_{H}}{r+r_{H}}$, such that the horizon is at $r_{*}=-\infty$ and the spacial infinity locates at $r_{*}=0$. The effective potential reads

$$
V(r)=\left(\lambda r^{2}-k\right)\left[m^{2}-5 \lambda / 4+\left(k / 4+\mu^{2}\right) r^{-2}\right] .
$$


For simplicity we will take $\lambda=1$. The potential is very similar to that of the massless scalar field perturbation described by the Klein-Gorden equation in the BTZ black hole on the brane where $V(r)=\left(\lambda r^{2}-k\right)\left[m^{2}-5 \lambda / 4+\left(k / 4+\mu^{2}\right) r^{-2}\right][20]$, which vanishes at the horizon but diverges at spatial infinity.

Adopting $x=1 / \cosh ^{2} r_{*}=\left(r^{2}-r_{H}^{2}\right) / r^{2}[20,21]$, and $\psi(r(x))=(1-x)^{1 / 4-\sqrt{m^{2}-1} / 2} x^{-i \omega / 2} y(x)$, we can transform Eq.(47) into a hypergeometric equation as Eq.(26) , but now with $a=\frac{1}{2}\left(1-\sqrt{m^{2}-1}-i \omega+i \mu\right), b=\frac{1}{2}\left(1-\sqrt{m^{2}-1}-\right.$ $i \omega-i \mu)$, and $c=1-i \omega$. To meet the boundary condition at the horizon $x=0$ where there are only ingoing waves, we choose the solution of the hypergeometric equation to be simply $y(x)={ }_{2} F_{1}(a, b, c ; x)$, so that

$$
\begin{aligned}
\psi & =(1-x)^{1 / 4-\sqrt{m^{2}-1} / 2} x^{-i \omega / 2}{ }_{2} F_{1}(a, b, c ; x), \\
H_{z z} & =(1-x)^{1 / 2-\sqrt{m^{2}-1} / 2} x^{-i \omega / 2}{ }_{2} F_{1}(a, b, c ; x) .
\end{aligned}
$$

We will check the restrictions of the boundary condition at spatial infinity. We write the asymptotical form of $H_{z z}$ at spatial infinity in the standard form Eq.(39). Using the transformation relation of hypergeometric function Eq. (29) we have, at spatial infinity,

$$
H_{z z} \sim G_{+} r^{-1+\sqrt{m^{2}-1}}+G_{-} r^{-1-\sqrt{m^{2}-1}}
$$

where

$$
G_{+}=\frac{\Gamma(c) \Gamma(c-a-b)}{\Gamma(c-a) \Gamma(c-b)}, \quad G_{-}=\frac{\Gamma(c) \Gamma(a+b-c)}{\Gamma(a) \Gamma(b)}
$$

When $m^{2}<2 \lambda$, it is easy to see that the boundary condition that $H_{z z}$ vanish at infinity is satisfied automatically, which is similar to what we observed in the fermonic perturbation. This means that there is no limit on the frequency of the perturbation, which can allow the perturbation with growing modes to exist and make the spacetime to be unstable.

When $m^{2} \geq 2 \lambda$, we need $G_{+}=0$ to ensure the vanishing of $H_{z z}$ at spatial infinity. Thus we should have $c-a$ or $c-b$ to be zero or negative integers, which leads to

$$
\omega= \pm \mu-i\left(2 n+1+\sqrt{m^{2} / \lambda-1}\right)
$$

where we have restored the $\lambda$. Notice that the imaginary part of the frequency is negative, which describes the decay mode of the perturbation. Thus the BTZ black string is stable in this case.

\section{Numerical Result of the Tensor Perturbation}

Now we try to solve the equation of the tensor perturbation Eq(44). It has to satisfy the boundary condition that $H_{t r}$ vanishes at spatial infinity.

From the asymptotic behavior Eq.(46), it is easy to see that the boundary condition can be satisfied automatically when $m^{2}<3 \lambda$. This again means that in this situation, there is no restriction on the frequency of the perturbation and even the growing mode is permitted which can make the spacetime unstable.

When $m^{2} \geq 3 \lambda$, we need to solve Eq. (44) to examine the perturbation behavior. This equation cannot be solved analytically, which requires numerical calculation. Considering that the spacetime has the AdS property, we will employ the numerical technique developed in [17] and applied in many AdS black hole spacetimes [19]. The radial equation Eq(44) keeps the same form under the transformation $r \rightarrow \alpha r, m \rightarrow m / \alpha$ and $\Omega \rightarrow \Omega / \alpha$. We will take $\lambda=1$ for simplicity. Taking $x=r^{-1}$, we can rewrite Eq.(44) in the form

$$
s(x) H_{t r, x x}+\frac{t(x)}{x-x_{H}} H_{t r, x}+\frac{u(x)}{\left(x-x_{H}\right)^{2}} H_{t r}=0,
$$

where $x_{H}=1 / r_{H}=1$, and

$$
\begin{aligned}
s(x)= & \left(2+m^{2}-\Omega^{2}\right) x^{6}+\left(4+2 m^{2}-2 \Omega^{2}\right) x^{5}-2\left(1+m^{2}\right) x^{3}-\left(1+m^{2}\right) x^{2}, \\
t(x)= & \left(2+m^{2}-\Omega^{2}\right)\left(x^{6}+x^{5}\right)-\left(7+2 m^{2}-5 \Omega^{2}\right)\left(x^{4}+x^{3}\right)-3\left(1+m^{2}\right)\left(x^{2}+x\right), \\
u(x)= & -\left(2+m^{2}-\Omega^{2}\right) x^{6}-\left(5+m^{2}-m^{4}+2 m^{2} \Omega^{2}-\Omega^{4}\right) x^{4} \\
& +2\left(7+2 m^{2}-m^{4}-3 \Omega^{2}+m^{2} \Omega^{2}\right) x^{2}-3-2 m^{2}+m^{4} .
\end{aligned}
$$


We can expand these functions around $x_{H}$, e.g. $s(x)=\sum_{n=0}^{\infty} s_{n}\left(x-x_{H}\right)^{n}$, and so does $t(x)$ and $u(x)$. $H_{t r}$ can also be written in the expansion form $H_{t r}=\sum_{n=0}^{\infty} a_{n}\left(x-x_{H}\right)^{n+\rho}$. Inserting all these expansions into Eq.(54), we can solve the leading order equation and find $\rho=-1 \pm \Omega / 2$. We will take the plus sign which stands for the in-going wave near the horizon. Equating the coefficients of $\left(x-x_{H}\right)^{n}$, we have the recursion relation for $a_{n}$ :

$$
a_{n}=-\frac{1}{P_{n}} \sum_{k=0}^{n-1}\left[(k+\rho)(k+\rho-1) s_{n-k}+(k+\rho) t_{n-k}+u_{n-k}\right] a_{k}
$$

where

$$
P_{n}=(n+\rho)(n+\rho-1) s_{0}+(n+\rho) t_{0}+u_{0}
$$

Considering the boundary condition that $\left.H_{t r}\right|_{x=0}=0$ at infinity, we require that $\sum_{n=0}^{\infty} a_{n}(\Omega, m)\left(-x_{H}\right)^{n}=0$, which is satisfied only for discrete values of $\Omega$. In the later discussion, we will take $\omega=i \Omega$. We need to find the zeros of $\sum_{n=0}^{\infty} a_{n}(\omega, m)\left(-x_{H}\right)^{n}=0$ in the complex $\omega$ plane. This is done by truncating the series after a large number of terms and computing the partial sum as a function of $\omega$. We can then find zeros of this partial sum, and check the accuracy by seeing how much the location of the zero changes as we go to higher partial sums.

To check the numerical scheme, we will first use it to calculate the scalar part perturbation, which can be compared with analytical results. For the scalar part, the corresponding $s(x)=4 x^{4}+8 x^{3}+4 x^{2}, t(x)=8 x^{4}+8 x^{3}$, and $u(x)=\left(1+4 \mu^{2}\right) x^{4}-\left(6-4 m^{2}+4 \mu^{2}+4 \Omega^{2}\right) x^{2}+5-4 m^{2}$. We truncate the infinite series at $N=200$ and get the numerical result listed in Table 1 . We find that the numerical calculation agrees well with the analytic solution, especially for the lower modes. This gives us confidence to use the numerical scheme to explore the tensor perturbation. Results for the tensor perturbation are shown in Table 2. It is interesting to find that for $m^{2} \geq 3 \lambda$, all modes have negative imaginary parts of QNM frequencies, which shows that in this case the spacetime is stable against tensor perturbation. Furthermore we observed that for lower modes the QNM frequency only has the imaginary part in the tensor perturbation and surprisingly its value satisfies $\omega=-i\left(2 n-1+\sqrt{m^{2}+1}\right), n=0,1,2 \ldots$

\begin{tabular}{ccc|rr}
\hline \hline$m^{2}$ & $\mu$ & $n$ & $\omega_{\text {num }}$ & $\omega_{\text {exact }}$ \\
\hline 2 & 0 & 0 & $-2.0000 i$ & $-2 i$ \\
& & 1 & $-4.0001 i$ & $-4 i$ \\
& & 2 & $-6.0002 i$ & $-6 i$ \\
& & 3 & $-8.0003 i$ & $-8 i$ \\
\hline 2 & 1 & 0 & $\pm 1.0000-2.0000 i$ & $\pm 1-2 i$ \\
& & 1 & $\pm 1.0000-4.0000 i$ & $\pm 1-4 i$ \\
& & 2 & $\pm 0.97-5.997 i$ & $\pm 1-6 i$ \\
\hline 3 & 0 & 0 & $-2.4140 i$ & $-2.41421 i$ \\
& & 1 & $-4.4142 i$ & $-4.41421 i$ \\
& & 2 & $-6.41 i$ & $-6.41421 i$ \\
\hline
\end{tabular}

Table 1. Comparison between the numerical result and the analytic result of $\omega$ for the scalar part perturbation.

\begin{tabular}{r|rrr|r}
\hline \hline$m^{2}$ & $\left.\omega\right|_{n=0}$ & $\left.\omega\right|_{n=1}$ & $\left.\omega\right|_{n=2}$ & $-1+\sqrt{m^{2}+1}$ \\
\hline 3.001 & $-1.0002500 i$ & $-3.0002500 i$ & $\pm 2 \times 10^{-7}-5.0002500 i$ & 1.0002500 \\
4 & $-1.236068 i$ & $-3.236068 i$ & $\pm 0.00007-5.236068 i$ & 1.2360680 \\
5.25 & $-1.5000000 i$ & $-3.5000000 i$ & $\pm 0.00004-5.5000000 i$ & 1.5 \\
6.25 & $-1.6925824 i$ & $-3.6925824 i$ & $\pm 0.00001-5.6925824 i$ & 1.6925824 \\
8.01 & $-2.0016662 i$ & $-4.0016662 i$ & $\pm 3 \times 10^{-8}-6.0016662 i$ & 2.0016662 \\
11.25 & $-2.5000000 i$ & $-4.5000000 i$ & $\pm 0.000001-6.5000000 i$ & 2.5 \\
16 & $-3.1231056 i$ & $-5.1231056 i$ & $\pm 4 \times 10^{-8}-7.1231056 i$ & 3.1231056 \\
19.25 & $-3.5000000 i$ & $-5.5000000 i$ & $\pm 4 \times 10^{-8}-7.5000000 i$ & 3.5 \\
\hline
\end{tabular}

Table 2. Quasinormal frequencies of tensor perturbation obtained through numerical calculation when $m^{2} \geq 3 \lambda$. 


\section{CONCLUSIONS AND DISCUSSIONS}

We have studied the stability of the BTZ black string against fermonic perturbation and gravitational perturbation. It is interesting to see that the BTZ black string can be dynamically stable provided that the $m^{2}$, which is determined by the compactification of the extra dimension, is over a threshold value, namely $\lambda, 2 \lambda, 3 \lambda$ respectively for fermonic perturbation, scalar part of the gravitational perturbation and the tensor perturbation. When $m^{2}$ is smaller than this threshold value, the perturbation can have a growing mode, which indicates that the BTZ black string can be unstable and pinch-off to form a black hole.

The situation that the stability and instability coexist in the dynamical system for the BTZ black string can be understood from thermodynamical arguments, if we compare the black string phase with the localized black hole phase with $\bar{m} A \rightarrow 0$. The entropy argument provided in [10] shows that the BTZ black string is not a privileged stable phase. Its entropy equals to that of the localized BTZ black hole with $\bar{m} A \rightarrow 0$. Our dynamical study supports this result.

It is of great interest to extend our discussion to the solution with localized black holes on the brane [10] where the geometry induced on the two-brane is described by $d s^{2}=-\left(\lambda r^{2}-k-2 \bar{m} A / r\right) d t^{2}+\left(\lambda r^{2}-k-2 \bar{m} A / r\right)^{-1} d r^{2}+r^{2} d \varphi^{2}$. The extra term $2 \bar{m} A / r$ came from the four-dimensional nature of the black hole. From the entropy argument it was argued that when $\bar{m} A$ is deviated from zero, entropies of black string and black hole will be nonzero and the black string entropy will exceed that of the black hole for small $\bar{m} A$. But for large $\bar{m} A$, the black string entropy will be lower. Thus the black string will first be stable and then become unstable and pinch-off to form a black hole [10]. The dynamical description of this process needs careful investigation and we will report its result elsewhere.

\section{Acknowledgments}

This work was partially supported by NNSF of China, Shanghai Education Commission and Shanghai Science and Technology Commission. B.W. would like to acknowledge helpful discussions with E. Abdalla and the associate programme in ICTP where his work was completed.

[1] R. Gregory and R. Laflamme, Phys. Rev. Lett. 70 2837(1993); R. Gregory and R. Laflamme, Nucl. Phys. B 428,399 (1994).

[2] R. Gregory and R. Laflamme, Nucl. Phys. B428, 399 (1994), arXiv: hep-th/9404071

[3] R. Gregory, Class. Quant. Grav. 17(2000), L125, arXiv: hep-th/0004101.

[4] T. Hirayama and G. Kang, Phys. Rev. D64, 064010 (2001), arXiv: hep-th/0104213

[5] T. Harmark, V. Niarchos and N. A Obers, Class. Quan. Grav. 24, R1 (2007).

[6] S. Gubser and I. Mitra, hep-th/0009126, S. Gubser and I. Mitra, J. High Energy Phys. JHEP08(2001)018.

[7] H.S. Reall, Phys. Rev. D 64044005 (2001); J. P. Gregory and S. F. Ross, Phys. Rev. D 64124006 (2001); T. Harmark, V. Niarchos and N. A. Obers, J. High Energy Phys. JHEP10(2005)045; J. Friess and S. Gubser, J. High Energy Phys. JHEP11(2005)040.

[8] J. Friess, S. Gubser and I. Mitra, Phys. Rev. D 72104019 (2005); D. Marolf and B. C. Palmer, Phys. Rev. D 70084045 (2004).

[9] X. He, S. B. Chen, B. Wang, R. G. Cai, C. Y. Lin, arXiv:0802.2449.

[10] R. Emparan, G. T. Horowitz, R. C. Myers, JHEP 0001 (2000) 021.

[11] M. Banados, C. Teitelboim and J. Zanelli, Phys. Rev. Lett. 69 (1992) 1849.

[12] Dieter R. Brill and John A. Wheeler, Rev. Mod. Phys. 29465 (1957).

[13] H. P. Nollert, Class. Quant. Grav. 16, R159 (1999); K. D. Kokkotas and B. G. Schmidt, Living Rev. Rel. 2 , 2 (1999).

[14] B. Wang, Braz. J. Phys. 35, 1029 (2005).

[15] I. S. Grandshteyn and I. M. Ryzhik, Table of Integrals, Series, and Products, (Academic Press, 1980).

[16] R. M. Wald, General Relativity, (The University of Chicargo Press, 1984).

[17] G. T. Horowitz and V. E. Hubeny, Phys. Rev. D62, 024027 (2000).

[18] Elcio Abdalla, Bin Wang, A. Lima-Santos and W.G. Qiu Phys. Lett. B538 (2002) 435, hep-th/0204030 E. Abdalla, K.H.C. Castello-Branco and A. Lima-Santos Phys. Rev. D66 (2002) 104018, hep-th/0208065

[19] B. Wang, C.Y. Lin, and E. Abdalla, Phys. Lett. B 481, 79 (2000); B. Wang, C.Y. Lin and C. Molina, Phys.Rev. D70 (2004) 064025.

[20] V. Cardoso and J. P. S. Lemos, Phys. Rev. D63, 124015 (2001), gr-qc/0101052

[21] Danny Birmingham, Phys. Rev. D64, 064024 (2001), hep-th/0101194. 